\title{
Enhanced Channel Access Estimation based Adaptive Control of Distributed Cognitive Radio Networks
}

\author{
Jong-Hong Park and Jong-Moon Chung \\ School of Electrical \& Electronic Engineering, Yonsei University \\ Seoul, Republic of Korea \\ [e-mail: jhwannabe@yonsei.ac.kr, jmc@yonsei.ac.kr] \\ *Corresponding author: Jong-Moon Chung
}

Received October 29, 2015; accepted January 12, 2016; published March 31, 2016

\begin{abstract}
Spectrum sharing in centrally controlled cognitive radio (CR) networks has been widely studied, however, research on channel access for distributively controlled individual cognitive users has not been fully characterized. This paper conducts an analysis of random channel access of cognitive users controlled in a distributed manner in a CR network. Based on the proposed estimation method, each cognitive user can estimate the current channel condition by using its own Markov-chain model and can compute its own blocking probability, collision probability, and forced termination probability. Using the proposed scheme, CR with distributed control (CR-DC), CR devices can make self-controlled decisions based on the status estimations to adaptively control its system parameters to communicate better.
\end{abstract}

Keywords: Cognitive radio, Blocking probability, Forced termination probability, Spectrum handoff, Spectrum sharing.

A preliminary version of this paper was presented at APIC-IST 2015 and was selected as an outstanding paper. This research was supported by Basic Science Research Program through the National Research Foundation of Korea (NRF) funded by the Ministry of Education, Science and Technology (No. 2013R1A1A2012082), the ICT R\&D program of MSIP/IITP [B0101-15-1276, Access Network Control Techniques for Various IoT Services] supervised by the Institute for Information \& communications Technology Promotion (IITP), and the ICT R\&D program of MSIP/IITP. [R0126-15-1009, Development of Smart Mediator for Mashup Service and Information Sharing among ICBMS Platform]. 


\section{Introduction}

In cognitive radio (CR) networks, each cognitive user, or secondary user, has a different channel access probability depending on the network topology to access licensed channels when unoccupied by primary users. Since a user with more neighbors experiences more severe competition in channel access, the events of blocking and collision occur more frequently. In wireless mesh networks, a node with a number of neighbors commonly has a crucial role to support in addition to its own communication needs, such as relaying/forwarding, however, it is rather difficult to be efficient in channel access without accurate channel access estimations.

Due to the importance of CR networks, studies on blocking probability and forced termination probability of cognitive users have been conducted. However most previous studies have analyzed the probability of the entire CR network characteristics. There are few modeling studies based on an individual cognitive user's performance point of view. Disadvantages of modeling of the overall network perspective is that it does not help individual CR nodes to make a decision on when is best to transmit. This paper proposes a channel access modeling scheme of cognitive users considering collisions between cognitive users from an individual cognitive user's point of view with distributed control in CR networks.

The contributions of this paper, compared with related published papers, are explained in the following.

Most papers which cover CR environments use a Markov-chain to analyze centralized CR networks [1]-[4]. Centralized models assume that there is a controller that monitors the entire channel and figures out the conditions of the primary and secondary CR channels.

In addition, the models in [1]-[4] do not consider collision events between cognitive users transmitting at the same time. In the proposed scheme, it is possible to analyze the collision probability of each cognitive user because it considers all possible collision events. As a result, the proposed model is able to accurately derive the individual channel access probability of cognitive users in distributed networks, and can estimate each individual cognitive user's blocking and forced termination probability by observing the CR user's channel conditions.

In [5] and [6], the active probability is used, where active is defined as a function of on-off control in sensor networks. Such approaches cannot characterize the performance when each cognitive user adjusts its own active probability value based on the condition of channel utilization. This is important because in a distributed CR network a CR user can modify its active probability by estimating channel conditions and controlling its transmission to satisfy its own communication needs.

Throughput of cognitive users is analyzed in [7], which is based on control of the arrival rate of all cognitive users. The scheme for adjusting the arrival rate of cognitive users in [7] is dependent on the number of channels unoccupied by primary users or other cognitive users. The method used in [7] is based on adjusting the arrival rate by the number of empty channels, which is inappropriate to adapt to distributed networks because this method is based on the control of the arrival rate in a centralized manner and does not consider the characteristics of a distributed CR network. In addition, since the approach in [7] uses a modeling scheme for the probability of selecting the appropriate channels, it cannot analyze important probabilities in CR networks such as blocking, collision, and forced termination. 
The proposed scheme can obtain the steady-state probability of channels looking at each cognitive user using a Markov-chain model, where the steady-state probability, blocking probability, collision probability, access probability, and forced termination probability of each cognitive user can be obtained accurately.

The remaining sections of this paper are organized as follows. In section II, the network model is introduced. Section III presents the performance analysis and section IV concludes the paper.

\section{System Model}

\subsection{Active Probability}

The active probability $p(k)(1 \leq k \leq N)$ is influenced by the number of cognitive users in the network $N$, which is a value that adjusts the likelihood of cognitive user $k$ to participate in the competition of channel access by sensing the channel status, which may be idle (unoccupied) or busy (occupied). Since the active probability of each cognitive user can be derived, a cognitive user can access the idle channel more effectively based on the network topology and channels situation (e.g., considering the number of active neighboring users).

\subsection{Markov Model for Distributed CR}

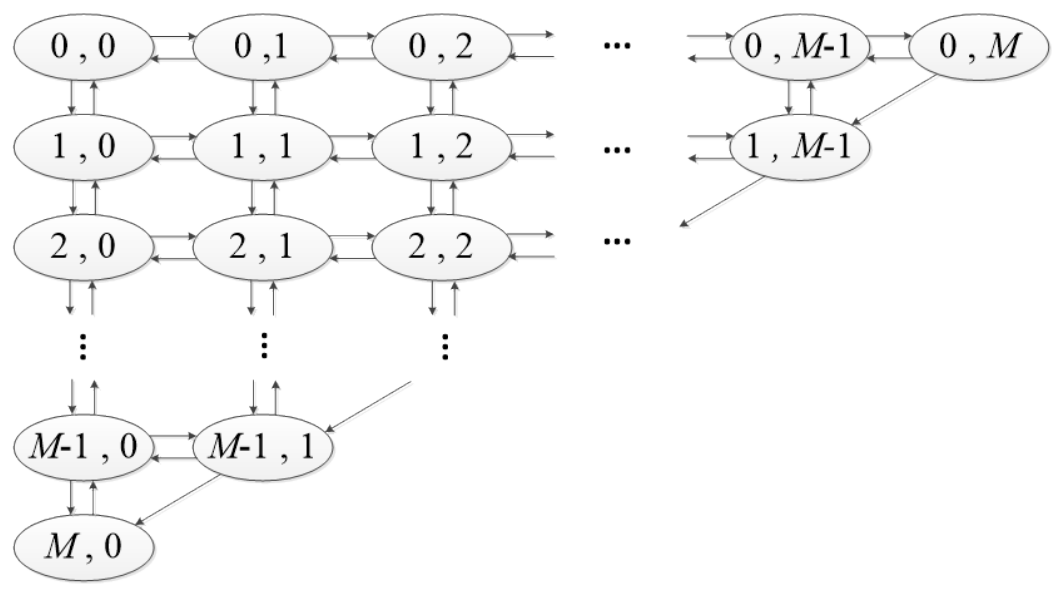

Fig. 1. Markov-chain model for a cognitive user

Fig. 1 presents the Markov-chain model of channel states for a cognitive user's perspective in the network with $M$ channels where spectrum handoff is possible. The number of all feasible states is $(M+1)(M+2) / 2$. In the integer pair $(i, j), i$ is the total number of primary users in channel use and $j$ is the total number of one-hop neighboring cognitive users using unoccupied channels by primary users. The arrivals of primary users and cognitive users are assumed Poisson processes with arrival rates $\lambda_{p}$ and $\lambda_{c}$, respectively. The service times are exponentially distributed with rates $\mu_{\mathrm{p}}$ and $\mu_{c}$, respectively [1]. 


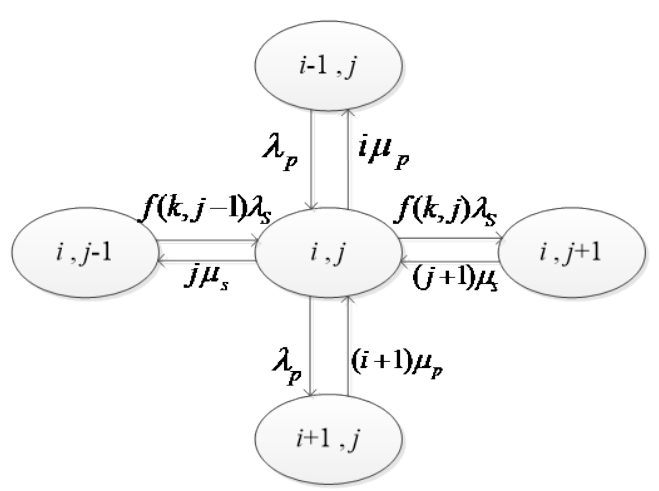

(a) $i+j<M$

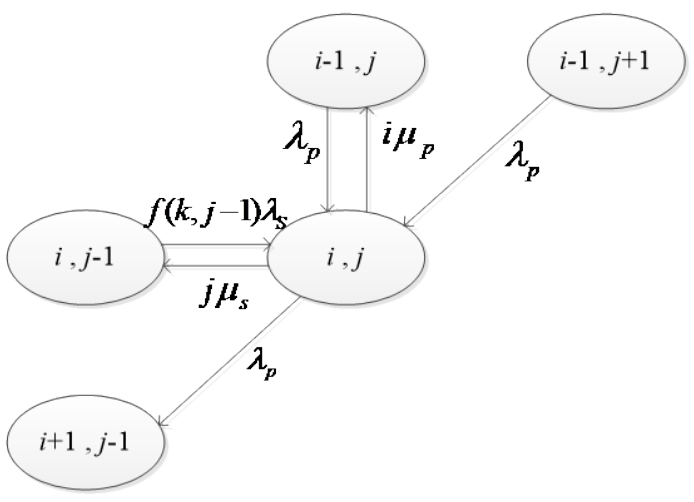

(b) $i+j=M$

Fig. 2. Markov-chain model for a cognitive user

The state transition diagram with spectrum handoff of cognitive user $k$ is depicted in Fig. 2. In state transition, $f(k, j)$ is a function of probability that one of the one-hop neighboring cognitive users of cognitive user $k$ accesses an unoccupied channel when $j$ neighboring cognitive users are already using the channels. This probability function can be expressed as follows

$$
f(k, j)=\left(\begin{array}{c}
\rho(k)-j \\
1
\end{array}\right) \widetilde{p}(k)(1-\widetilde{p}(k))^{\rho(k)-j}
$$

where $\rho(k)$ is the total number of one-hop neighboring cognitive users of $k$, and $\tilde{p}(k)$ is the average of active probabilities of one-hop neighboring cognitive users of $k$.

For $(i, j) \in \Phi_{k}, \phi_{k}(i, j)$ is the indicator function of cognitive user $k$ in Fig. 2, where $\Phi_{k}$ is the set of feasible states. The indicator function of user $k$ can be represented as follows.

$$
\phi_{k}(i, j)= \begin{cases}1 & \text { if }(i, j) \in \Phi_{k} \\ 0 & \text { otherwise }\end{cases}
$$

The balance equation of Fig. 2 (a) can be represented as (3) where $\pi_{k}(i, j)$ is the state probability of state $(i, j)$ of cognitive user $k$.

$$
\begin{aligned}
& \left\{i \mu_{p}+f(k, j) \lambda_{s}+\lambda_{p}+j \mu_{s}\right\} \pi_{k}(i, j) \phi_{k}(i, j) \\
& =\lambda_{p} \pi_{k}(i-1, j) \phi_{k}(i-1, j)+(j+1) \mu_{s} \pi_{k}(i, j+1) \phi_{k}(i, j+1) \\
& \quad+(i+1) \mu_{p} \pi_{k}(i+1, j) \phi_{k}(i+1, j)+f(k, j-1) \lambda_{s} \pi_{k}(i, j-1) \phi_{k}(i, j-1)
\end{aligned}
$$

Similarly, the balance equation of Fig. 2 (b) can be expressed as follows. 


$$
\begin{aligned}
& \left\{i \mu_{p}+\lambda_{p}+j \mu_{s}\right\} \pi_{k}(i, j) \phi_{k}(i, j) \\
& =\lambda_{p} \pi_{k}(i-1, j) \phi_{k}(i-1, j)+\lambda_{p} \pi_{k}(i-1, j+1) \phi_{k}(i-1, j+1) \\
& \quad+f(k, j-1) \lambda_{s} \pi_{k}(i, j-1) \phi_{k}(i, j-1)
\end{aligned}
$$

Considering the effect of spectrum handoff, the number of all feasible states is $(M+1)(M+2) / 2$, and the sum of all feasible states is 1 , as presented in (5). By solving the balance equations, all state probabilities of a cognitive user can be found.

$$
\sum_{i=0}^{M} \sum_{j=0}^{M} \pi_{k}(i, j) \phi_{k}(i, j)=1
$$

\subsection{Blocking Probability, Collision Probability and Access Probability}

In CR networks, the blocking of cognitive users occurs when the state of a cognitive user becomes active and a cognitive user starts sensing the channels, but the channels are occupied by primary users or its one-hop neighboring cognitive users. The blocking probability of cognitive user $k\left(P_{B}(k)\right)$, can be obtained by multiplying the active probability of $k(p(k))$ and applying the summation of all full occupied states $\left(\hat{P}_{B}(k)\right)$. Therefore the blocking probability of cognitive user $k$ can be calculated from the Markov-chain model as follows.

$$
P_{B}(k)=\sum_{i+j=M} \pi_{k}(i, j) p(k)=\hat{P}_{B}(k) p(k)
$$

If the active probability of cognitive user $k$ is $1, P_{B}(k)$ equals $\hat{P}_{B}(k)$.

For collision-free channel access for cognitive user $k$, at least one or more channels need to be idle, and the channels of user $k$ 's one-hop neighboring cognitive users must not be fully used. Fig. 3 illustrates this 'hidden terminal problem' case.

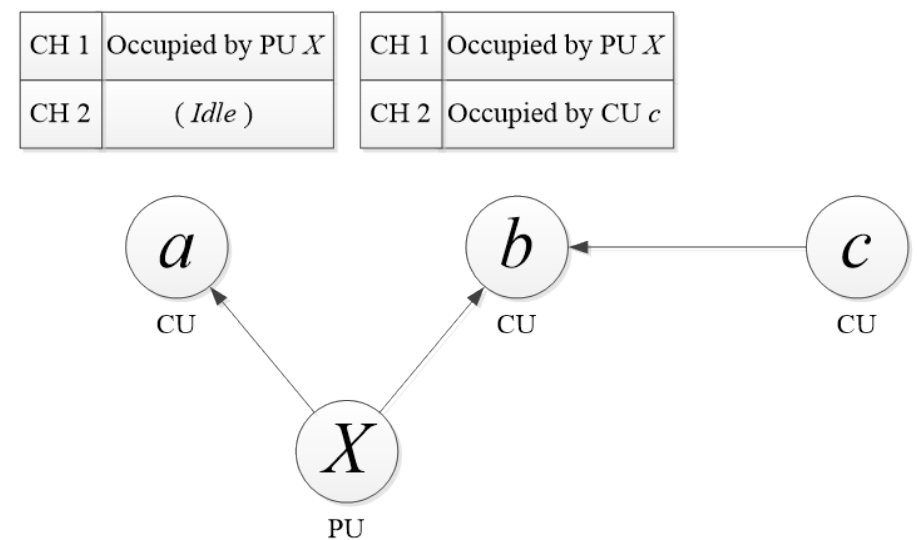

Fig. 3. Hidden terminal problem 
In Fig. 3, when a cognitive user $a$ senses the state of two channels, it draws inference that only $\mathrm{CH} 1$ is occupied by primary user (PU) $X$, and $\mathrm{CH} 2$ is not used by any other users. However, from the cognitive user (CU) $b$ 's perspective, it detects that $\mathrm{CH} 1$ is used by $\mathrm{PU} X$, but $\mathrm{CU} b$ can sense that $\mathrm{CH} 2$ is used by its one-hop neighboring $\mathrm{CU} c$. In this situation, if $\mathrm{CU}$ $a$ broadcasts using $\mathrm{CH} 2$, a collision occurs at CU $b$. Therefore, collision of a cognitive user trying to access a channel occurs when the channels are fully used by at least one of the one-hop neighboring users.

For successful channel access of a cognitive user, the blocking and collision should not occur at the same time when a cognitive user is active. The representation of successful channel access probability of a cognitive user is presented in (8). $P_{\text {Nonblock }}(k)$ in (7) is the non-blocking probability of all one-hop neighboring users of cognitive user $k$, when the number of one-hop neighboring users of $k$ is $n\left(l_{1}, l_{2}, \ldots, l_{n}\right)$.

$$
\begin{aligned}
P_{\text {Nonblock }}(k) & =\left(1-\sum_{i+j=M} \pi_{l_{1}}(i, j)\right)\left(1-\sum_{i+j=M} \pi_{l_{2}}(i, j)\right) \cdots\left(1-\sum_{i+j=M} \pi_{l_{n}}(i, j)\right) \\
& =\left(1-\hat{P}_{B}\left(l_{1}\right)\right)\left(1-\hat{P}_{B}\left(l_{2}\right)\right) \cdots\left(1-\hat{P}_{B}\left(l_{n}\right)\right) \\
& =\prod_{l \in \Psi(k)}\left(1-\hat{P}_{B}(l)\right) \\
& P_{A}(k)=\left(1-\hat{P}_{B}(k)\right) P_{\text {Nonblock }}(k) p(k)
\end{aligned}
$$

In (7), $\Psi(k)$ is the set of all one-hop neighboring users of cognitive user $k$.

The collision probability of cognitive user $k$ can be obtained as (9) assuming that the channel environment of primary users' usage is equal to all cognitive users in the network.

$$
P_{C}(k)=\left(1-\hat{P}_{B}(k)\right)\left(1-P_{\text {Nonblock }}(k)\right) p(k)
$$

The idle (i.e., the non-active) probability of cognitive user $k$ can be expressed as (10) based on the fact that the probability sum of all possible outcomes is equal to 1 .

$$
\begin{aligned}
P_{I}(k) & =1-p(k) \\
& =1-p(k)\left[\hat{P}_{B}(k)+\left(1-\hat{P}_{B}(k)\right)\right] \\
& =1-p(k)\left[\hat{P}_{B}(k)+\left(1-\hat{P}_{B}(k)\right)\left\{P_{\text {Nonblock }}(k)+\left(1-P_{\text {Nonblock }}(k)\right)\right\}\right] \\
& =1-p(k) \hat{P}_{B}(k)-p(k)\left(1-\hat{P}_{B}(k)\right) P_{\text {Nonblock }}(k)-p(k)\left(1-\hat{P}_{B}(k)\right)\left(1-P_{\text {Nonblock }}(k)\right) \\
& =1-P_{B}(k)-P_{A}(k)-P_{C}(k)
\end{aligned}
$$

\subsection{Forced Termination Probability and Throughput}

Another important issue to analyze in distributed CR networks is forced termination. A cognitive user should vacate using a channel if a licensed primary user arrives. If there is another channel, a cognitive user can perform spectrum handoff. However, if not, forced termination occurs. In other words, the forced termination probability is the probability that a cognitive user already accessing and using a channel is forced to disconnect the service 
because of the primary user's appearance and has no other available channel to conduct handoff to. The forced termination probability can be obtained from the Markov-chain model of one-hop neighboring cognitive users. In the Markov-chain model of cognitive user $k$, the forced termination probability can be derived by subtracting the probability that forced termination will not occur from the access probability of cognitive user $k$, which results in the form of

$$
P_{F}(k)=\frac{\lambda_{p}}{\lambda_{s}} \frac{P_{A}(k)-P_{\text {Fnot }}(k)}{P_{A}(k)}
$$

where $P_{\text {Fnot }}(k)$ is the probability that successfully channel access of cognitive user $k$ will not result in forced termination because there is another vacant channel to perform spectrum handoff, which is represented in (12).

$$
P_{\text {Fnot }}(k)=\prod_{l \in \Psi(k)}\left(1-P_{B}(l)\right)-\prod_{l \in \Psi(k)}\left\{\left(1-P_{B}(l)\right)-\sum_{i+j=M-1} \frac{1}{j+1} \pi_{l}(i, j)\right\}
$$

Assuming that all cognitive users have an identical transmission rate, normalized to 1 bit per unit time, each cognitive user's throughput can be expressed as (13).

$$
S(k)=\frac{P_{A}(k)}{p(k)}\left(1-P_{F}(k)\right) \lambda_{s}\left(\frac{\mu_{s}}{1-P_{F}(k)}\right)^{-1}
$$

\section{Simulation \& Analysis}

In this section, by comparing the developed analytical results to the simulation results, the accuracy of the proposed modeling scheme, CR with distributed control (CR-DC), is demonstrated. The example networks of [8] are used as CR networks as illustrated in Fig. 4. In Fig. 4 (a), for example, the nodes 5 and 10 who have relatively a large number of one-hop neighboring users have a larger value of blocking and collision probability. As a result, they have less channel access probability. On the contrary to this, cognitive users such as 1,7 , and 8 who have a relatively small number of neighboring users have a bigger chance to access the channel. The reason for using Fig. 4 is that these topologies are suitable to apply to distributed CR networks because they are representatives of (a) simple and (b) complex wireless mesh networks. In addition, because the nodes in the network topology have a different number of one-hop neighboring nodes, they are used to analyze the accuracy of the proposed modeling scheme in this paper. Each node is assumed to be a cognitive user in the example networks and $M$ is set to 2 . 


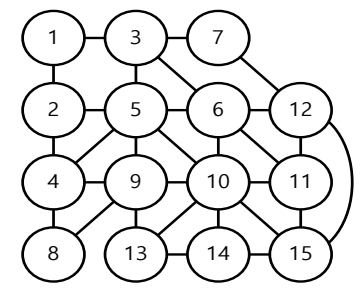

(a)

(b)

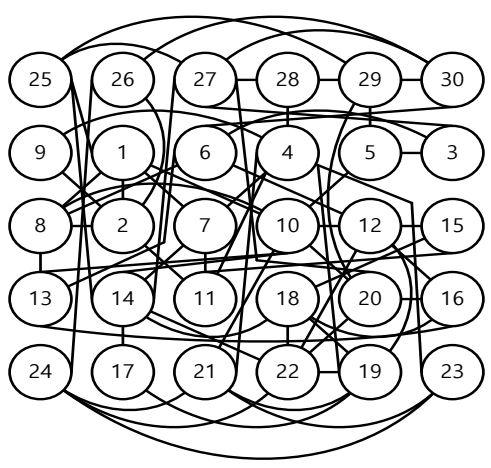

Fig. 4. Example networks presented in [8]

The access probability, blocking probability and collision probability based on the arrival rate of the primary users are analyzed for 15-node networks in Fig. 5. It is shown that the proposed CR-DC scheme provides an accurate performance compared to actual cognitive users in distributed cognitive networks.

The performance comparison with the centralized modeling algorithm from a position of channels centralized [2] is presented in Fig. 6. For accurate comparison with CR-DC, the number of sub-channels ( $N$ in [2]) is 1 and the number of channels ( $M$ in [2]) is 2. The performance of average throughput of each cognitive user in [2] does not consider the collisions between cognitive users. In addition, throughput analysis in [2] does not consider the topology of the network, therefore, throughput is only dependent on the number of channels regardless of the number of nodes in the network. Consequently there is a difference between the throughput analysis of [2] and simulation results in distributed CR networks. On the other hand, the proposed CR-DC considers the collisions between cognitive users and can precisely analyze the throughput of each cognitive user in a distributed CR network by taking into account the topology of the network.

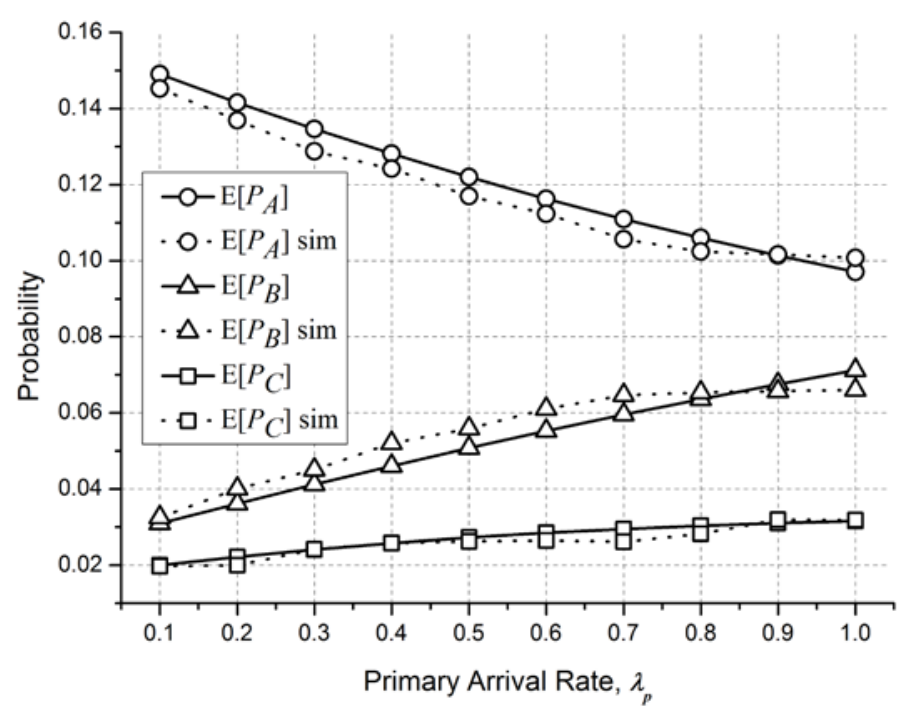

Fig. 5. Access probability, blocking probability and collision probability 


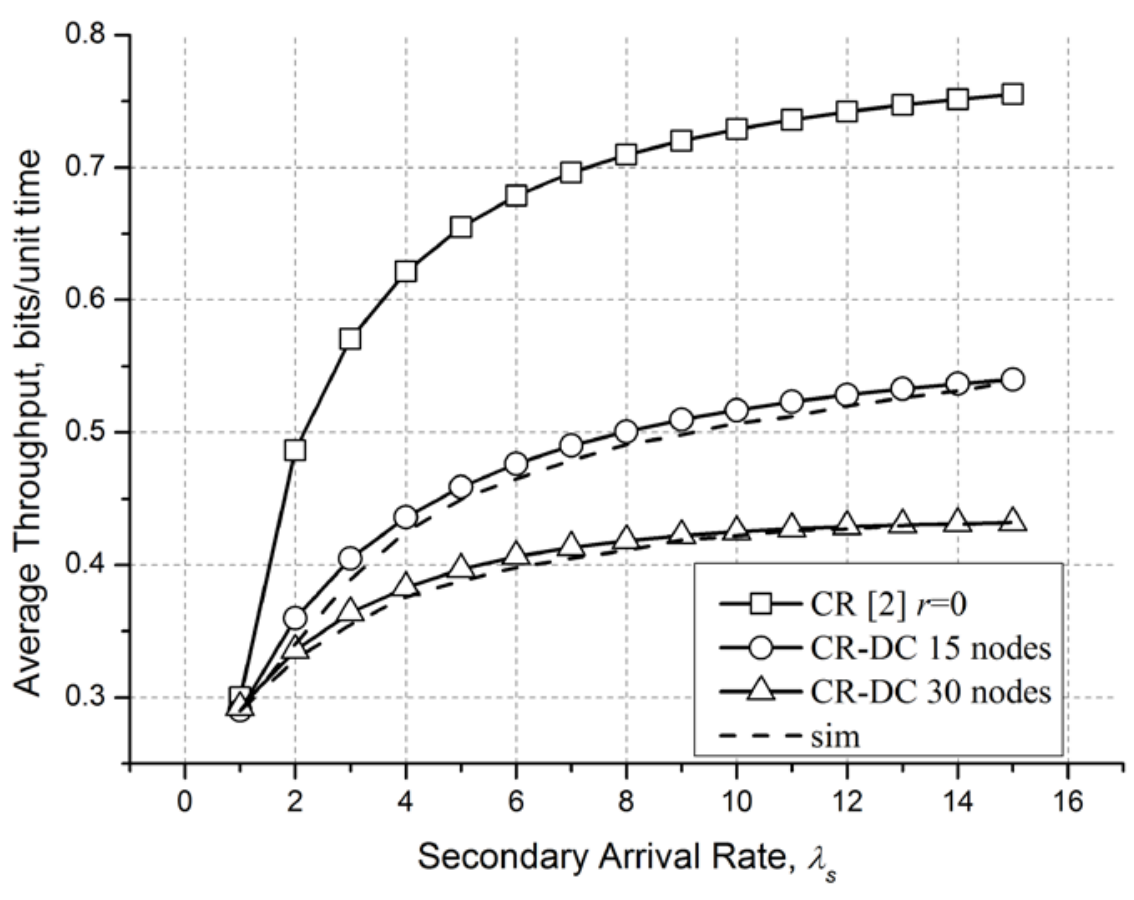

Fig. 6. Throughput comparison between the centralized modeling and CR-DC

\section{Conclusion}

The proposed CR-DC scheme can be used to obtain the steady-state probability of channels looking at each cognitive user by using a Markov-chain model. Through the steady-state probability, the blocking probability, collision probability, access probability, and forced termination probability of each cognitive user can be obtained accurately for a distributed CR network. Using the computed access probability of each cognitive user in the random channel access environment, the performance can be accurately obtained and using this information the cognitive users in the network can make self-controlled decisions (based on the status estimations) to adaptively control their system parameters to communicate better.

\section{References}

[1] X. Zhu, L. Shen, and T.-S. P. Yum, "Analysis of Cognitive Radio Spectrum Access with Optimal Channel Reservation,” IEEE Commun. Lett., vol. 11, no. 4, pp. 304-306, Apr. 2007. Article (CrossRef Link).

[2] W. Ahmed, J. Gao, H. A. Suraweera, and M. Faulkner, "Comments on "Analysis of Cognitive Radio Spectrum Access with Optimal Channel Reservation,” IEEE Trans. Wirel. Commun., vol. 8, no. 9, pp. 4488-4491, Sep. 2009. Article (CrossRef Link).

[3] J. Martinez-Bauset, V. Pla, and D. Pacheco-Paramo, "Analysis of Cognitive Radio Spectrum Access with Optimal Channel Reservation,” IEEE Commun. Lett., vol. 13, no. 10, p. 739, Oct. 2009. Article (CrossRef Link). 
[4] J.-M. Chung, C. H. Kim, J.-H. Park, J. Shin, and D. Kim, "Optimal Channel Allocation for Cognitive Radios,” IET Electron. Lett., vol. 46, no. 11, pp. 802-803, May 2010. Article (CrossRef Link).

[5] O. B. Akan, O. B. Karli, and O. Ergul, "Cognitive Radio Sensor Network,” IEEE Netw., vol. 23, no. 4, pp. 34-40, Jul/Aug. 2009. Article (CrossRef Link).

[6] X. Li, D. Wang, J. McNair, and J. Chen, "Residual Energy Aware Channel Assignment in Cognitive Radio Sensor Network,” in Proc. of IEEE WCNC`11, pp. 398-403, Cancun, Mexico, Mar. 2011. Article (CrossRef Link).

[7] S. Srinivasa and S. A. Jafar, "How Much Spectrum Sharing is Optimal in Cognitive Radio Networks?,” IEEE Trans. Wirel. Commun., vol. 7, no. 10, pp. 4010-4018, Oct. 2008. Article (CrossRef Link).

[8] G. Wang and N. Ansari, "Optimal Broadcast Scheduling in Packet Radio Networks Using Mean Field Annealing,” IEEE J. Sel. Areas Commun., vol. 15, no. 2, pp. 250-260, Feb. 1997. Article (CrossRef Link).

[9] J.-H. Park, Y. Nam, and J.-M. Chung, "Analysis of Channel Access with Spectrum Handoff in Cluster Based Cognitive Radio Sensor Networks,” in Proc. of ICTC'13, pp. 232-233, Jeju, Republic of Korea, Oct. 2013. Article (CrossRef Link).

[10] M. NoroozOliaee, B. Hamdaoui, X, Cheng, T. Znati, and M. Guizani, "Analyzing Cognitive Network Access Efficiency Under Limited Spectrum Handoff Agility,” IEEE Trans. Veh. Technol., vol. 63, no. 3, pp. 1402-1407, Mar. 2014. Article (CrossRef Link).

[11] "IEEE P802.22/D1.0 Draft Standard for Wireless Regional Area Networks Part 22: Cognitive Wireless RAN Medium Access Control (MAC) and Physical Layer (PHY) Specifications: Policies and Procedures for Operation in the TV Bands," Apr. 2008. Article (CrossRef Link).

[12] C. R. Stevenson, G. Chouinard, Z. Lei, W. Hu, S. J. Shellhammer, and W. Caldwell, "IEEE 802.22 The First Cognitive Radio Wireless Regional Area Network Standard,” IEEE Commun. Mag., vol. 47, no. 1, pp. 130-138, Jan. 2009. Article (CrossRef Link).

[13] G. A. Shah and O. B. Akan, "Cognitive Adaptive Medium Access Control in Cognitive Radio Sensor Networks,” IEEE Trans. Veh. Technol., vol. 64, no. 2, pp. 757-767, Feb. 2015. Article (CrossRef Link).

[14] M. Ghaznavi and A. Jamshidi, "A Reliable Spectrum Sensing Method in the Presence of Malicious Sensors in Distributed Cognitive Radio Network,” IEEE Sens. J., vol. 15, no. 3, pp. 1810-1816, Mar. 2015. Article (CrossRef Link).

[15] M. F. Kader, Asaduzzaman, and M. M. Hoque, "Hybrid Spectrum Sharing with Cooperative Secondary User Selection in Cognitive Radio Networks,” KSII Trans. Internet Inf. Syst., vol. 7, no. 9, pp. 2081-2100, Sep. 2013. Article (CrossRef Link).

[16] G. P. Joshi, S. Y. Nam, S. Acharya, and S. W. Kim, "Analysis of Channel Access Delay on CR-MAC Protocol for Ad Hoc Cognitive Radio Wireless Sensor Networks without a Common Control Channel,” KSII Trans. Internet Inf. Syst., vol. 8, no. 3, pp. 911-923, Mar. 2014.

Article (CrossRef Link). 


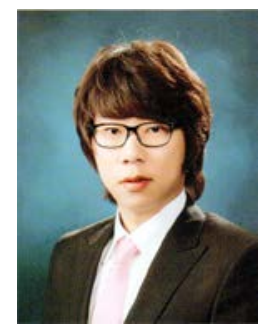

Jong-Hong Park received his B.S. degree from the School of Electrical and Electronic Engineering, Yonsei University, Seoul, Republic of Korea, in 2010. He is currently working towards a Ph.D. degree in the School of Electrical and Electronic Engineering and is also a research member of the Communications and Networking Laboratory (CNL) at Yonsei University. His research focuses on IoT device's cognitive radio (CR) networks, augmented reality (AR), and cloud computing.

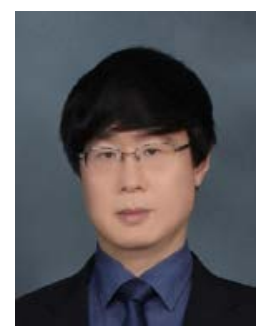

Dr. Jong-Moon Chung received B.S. and M.S. degrees in electronic engineering from Yonsei University, Seoul, Korea, in 1992 and 1994, respectively, and a Ph.D. degree in electrical engineering from the Pennsylvania State University, University Park, PA, USA, in 1999. Since 2005, he has been a Professor in the School of Electrical \& Electronic Engineering, Yonsei University, Seoul, Republic of Korea (ROK). From 1997 to 1999, he served as an Assistant Professor and Instructor in the Department of Electrical Engineering, Pennsylvania State University, University Park Campus, PA, USA. From 2000 to 2005, he was with the School of Electrical \& Computer Engineering, Oklahoma State University (OSU), Stillwater, OK, USA as a Tenured Associate Professor and Director of the OCLNB and ACSEL labs. His research is in the area of smartphone design, network scheduler design, M2M, IoT, AR, CR, SDN, NFV, MANET, VANET, WSN, satellite \& mobile communications, and broadband QoS networking. In 2000 he received the First Place Outstanding Paper Award at the IEEE EIT 2000 conference. In 2003 and 2004, respectively, he received the Distinguished Faculty Award and the Technology Innovator Award, both from OSU. As an Associate Professor at OSU, in October 2005, he received the Regents Distinguished Research Award and in September the same year he received the Halliburton Outstanding Young Faculty Award. In 2008 he received the Outstanding Accomplishment Professor Award from Yonsei University. In 2012 he received the ROK Defense Acquisition Program Administration (DAPA) Award. $\mathrm{He}$ is a member of the IET and IEICE and a life member of the HKN, KIIS, IEIE, and KICS. He has served as the General Co-Chair of IEEE MWSCAS 2011, Local Chair and TPC Co-Chair of IEEE VNC 2012, and Local Chair of IEEE WF-IoT 2014. He is also an Editor of the IEEE Transactions on Vehicular Technology. 